\title{
Influence of the stiffness coefficient of an elastic foundation on the frequency of free oscillations of polyethylene pipes in a semi- underground laying
}

\author{
Vladimir Sokolov, Igor Razov* and Sviatoslav Volinets \\ Industrial University of Tyumen, Volodarskogo str.38, Tyumen, 625001, Russia
}

\begin{abstract}
The article is about the study of the frequencies of free oscillations of a large-diameter polyethylene gas pipeline with a semiunderground installation, which carried out on the basis of the solution for a semi-underground thin-walled steel pipeline of large diameter. As a result of oscillations, the cross section of the pipeline is deformed, but it strongly depends on the geometrical and mechanical parameters set, for example, on the stiffness coefficient of the elastic medium
\end{abstract}

The need to ensure the reliability of trunk pipelines is caused by their operation in various climatic and geological conditions. There are several types of laying pipelines: aboveground, above ground, semi underground, underground and underwater. Each of them in the calculation requires the choice of the correct design scheme and the collection of loads. Until recently, pipelines in Russia were calculated from the standpoint of the classical rod theory, according to Building regulations 2.05.06. - $85 *$ and Set of Building Rules 36.13330.2012 - Trunk pipelines. However, the core model is only suitable for calculating thick-walled pipes with a ratio of wall thickness $h$ to radius $\mathrm{R}$ more than $1 / 20$ $(1 / 10,1 / 5$, etc.). Due to the use of modern thin-walled pipes with a diameter of more than 1000 millimeters in construction, this approach is wrong, because the core model does not take into account the effect of internal working pressure on the dynamic characteristics and stability of the pipeline. The use of a thin-walled cylindrical shell as the base design model will allow for the above-mentioned non-observance. Consequently, the further solutions obtained will be based on a geometrically non-linear variant of a semi-momentless theory of cylindrical shells. The solutions obtained make it possible to most fully take into account the influence of the internal pressure, the geometric characteristics, the longitudinal compressive force, the flow of the flowing fluid, and the influence of the surrounding elastic medium [1-5].

In this paper, we consider the underground type of laying gas pipelines of large diameter; therefore, it is necessary to investigate the effect of the soil pressure surrounding the pipeline and the effect of its frequency and the shape of free vibrations.

\footnotetext{
* Corresponding author: razovio@tyuiu.ru
} 
The study is carried out on the basis of the solution obtained in [5] for a semiunderground thin-walled steel pipeline of large diameter:

$$
\omega_{m n}^{2}=\frac{\lambda_{n}^{4}+m^{4}\left(m^{2}-1\right)\left(m^{2}-1-\gamma^{*}+p^{*}\right)+k^{*} m^{4}-\lambda_{n}^{4} m^{4} P / n^{2}}{\rho^{*} R \cdot h\left(\lambda_{n}^{2} h_{\tilde{v}}+m^{2}+m^{4}\right)},
$$

where $\lambda_{n}=\frac{n \pi R}{L \sqrt{h_{\tilde{v}}}}, \quad p^{*}=p_{0} \frac{R}{E h h_{v}^{2}}, \quad \rho^{*}=\rho_{0} \frac{R}{E h h_{v}^{2}}, \quad k^{*}=\frac{R^{2} k}{E h h^{2}}, \rho-$ shell material density; $\mathrm{R}$ is the radius of the cross section; $\mathrm{n}$ and $\mathrm{m}$ are wave numbers in the longitudinal and circumferential direction; $\mathrm{L}$ is the length of the shell; $\mathrm{p}_{0}$ is the internal working pressure; $\mathrm{h}$ is the shell thickness; $\mathrm{k}$ is the stiffness coefficient of the elastic medium; $h_{v}$ parameter of the relative thickness of the shell:

$$
h_{\tilde{v}}=\frac{h}{R \sqrt{12\left(1-v^{2}\right)}}
$$

where $v$ - Poisson's ratio; $\mathrm{E}$ is the elastic modulus of the shell material.

The study of the frequencies of free oscillations of a large-diameter polyethylene gas pipeline with a semi-underground installation was carried out with a pipe size $1200 \times 57,2$ millimetersand with a nominal pressure of $0.8 \mathrm{MPa}$, the ratio of length to radius $\mathrm{L} / \mathrm{R}=15$, the parameter of the longitudinal compressive force $\mathrm{P}=0.3$, and the stiffness coefficient of the elastic medium k varying in the range from 1 to $30 \mathrm{MN} / \mathrm{m} 3$. The obtained frequencies and forms of free oscillations are summarized in tables 1.2 and illustrated in the charts of figures 1-4.

Table 1. Dependence of frequencies of free oscillations of a semi-underground polyethylene gas pipeline PE100 on the value of the coefficient

\begin{tabular}{|c|c|c|c|c|c|c|}
\hline \multirow{3}{*}{$\left.\right|_{k} ^{n, m}$} & \multicolumn{6}{|c|}{ Polyethylene pipe PE100, 1200x57,2mm; L/R=15; P=0,3; $p_{0}=0,8 \mathrm{MПa}$} \\
\hline & \multicolumn{3}{|c|}{$n=1$} & \multicolumn{3}{|c|}{$n=2$} \\
\hline & $\mathbf{m}=1$ & $\mathbf{m}=2$ & $\mathbf{m}=\mathbf{3}$ & $\mathbf{m}=1$ & $\mathrm{~m}=2$ & $\mathbf{m}=\mathbf{3}$ \\
\hline 1 & 2,24 & 2,23 & 3,42 & 6,33 & 2,23 & 2,43 \\
\hline 3 & 3,13 & 3,72 & 4,67 & 6,59 & 3,66 & 3,99 \\
\hline 5 & 3,82 & 4,76 & 5,65 & 6,83 & 4,67 & 5,09 \\
\hline 10 & 5,15 & 6,71 & 7,56 & 7,41 & 6,55 & 7,13 \\
\hline 20 & 7,10 & 9,44 & 10,38 & 8,44 & 9,22 & 10,04 \\
\hline 30 & 8,62 & 11,56 & 12,59 & 9,36 & 11,27 & 12,28 \\
\hline 40 & 9,91 & 13,34 & 14,46 & 10,20 & 13,01 & 14,17 \\
\hline 50 & 11,05 & 14,91 & 16,11 & 10,90 & 14,54 & 15,84 \\
\hline
\end{tabular}
of the elastic medium $k$, for different values of the wave numbers $m$ and $n$. 
$\omega_{m n^{2}} H z$

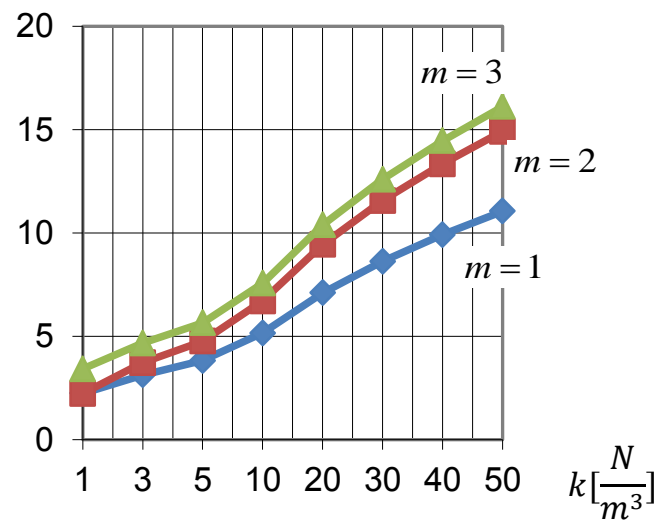

Fig. 1 - Dependence of frequencies of free oscillations of a semi-underground PE 100 gas pipeline on the value of the coefficient of the elastic medium $\mathrm{k}$, with $\mathbf{n}=\mathbf{1}$

$$
\omega_{m n^{2}} \mathrm{~Hz}
$$

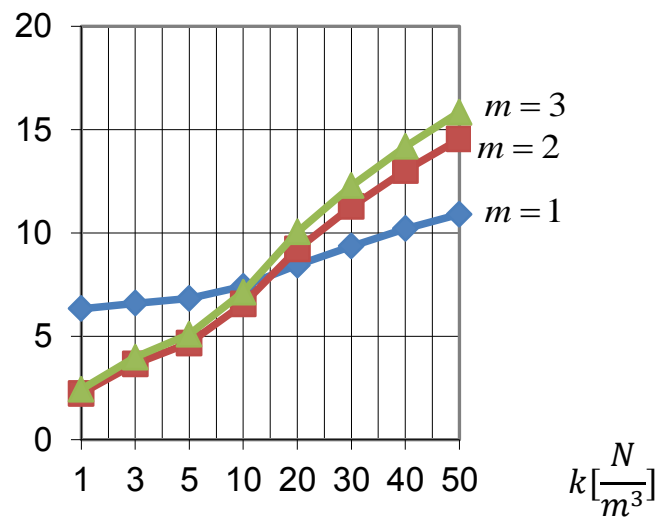

Fig. 2 - Dependence of frequencies of free oscillations of a semi-underground PE 100 gas pipeline on the value of the coefficient of the elastic medium $\mathrm{k}$, with $\mathbf{n}=\mathbf{2}$

Table 2. Dependence of frequencies of free oscillations of a semi-underground polyethylene gas pipeline PE100 on the value of the coefficient of the elastic medium $k$, for different values of the wave numbers $m$ and $n$.

\begin{tabular}{|c|c|c|c|c|c|c|}
\hline & \multicolumn{6}{|c|}{ Polyethylene pipe PE100, 1200x57,2mm; L/R=15; P=0,3; $p_{0}=0,8 \mathrm{M \Pi a}$} \\
\hline & \multicolumn{3}{|c|}{$n=1$} & \multicolumn{3}{|c|}{$n=2$} \\
\hline$k \quad\left[H / M^{3}\right]$ & $\mathbf{m}=1$ & $\mathbf{m}=\mathbf{2}$ & $\mathbf{m}=\mathbf{3}$ & $\mathbf{m}=1$ & $\mathrm{~m}=2$ & $\mathbf{m}=\mathbf{3}$ \\
\hline 1 & 2,24 & 2,23 & 3,42 & 6,33 & 2,23 & 2,43 \\
\hline 3 & 3,13 & 3,72 & 4,67 & 6,59 & 3,66 & 3,99 \\
\hline 5 & 3,82 & 4,76 & 5,65 & 6,83 & 4,67 & 5,09 \\
\hline 10 & 5,15 & 6,71 & 7,56 & 7,41 & 6,55 & 7,13 \\
\hline 20 & 7,10 & 9,44 & 10,38 & 8,44 & 9,22 & 10,04 \\
\hline 30 & 8,62 & 11,56 & 12,59 & 9,36 & 11,27 & 12,28 \\
\hline 40 & 9,91 & 13,34 & 14,46 & 10,20 & 13,01 & 14,17 \\
\hline 50 & 11,05 & 14,91 & 16,11 & 10,90 & 14,54 & 15,84 \\
\hline
\end{tabular}




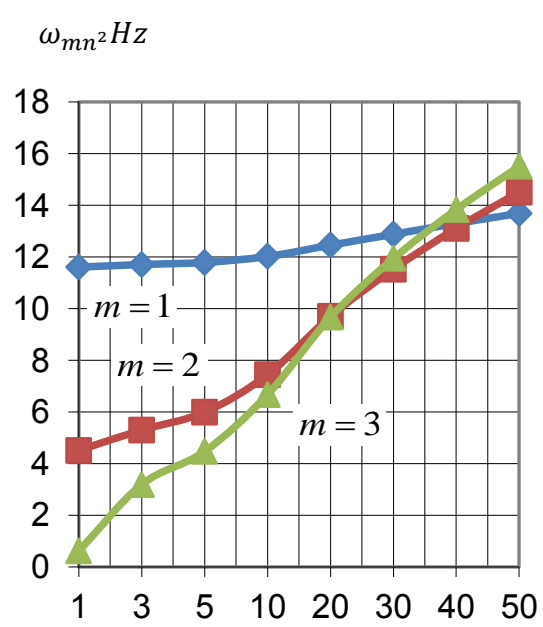

Fig. 3 - Dependence of frequencies of free oscillations of a semiunderground $\mathrm{PE} 100$ gas pipeline on the value of the coefficient of the elastic medium $\mathrm{k}$, with $\mathbf{n}=\mathbf{3}$

$$
\omega_{m n^{2}} \mathrm{~Hz}
$$

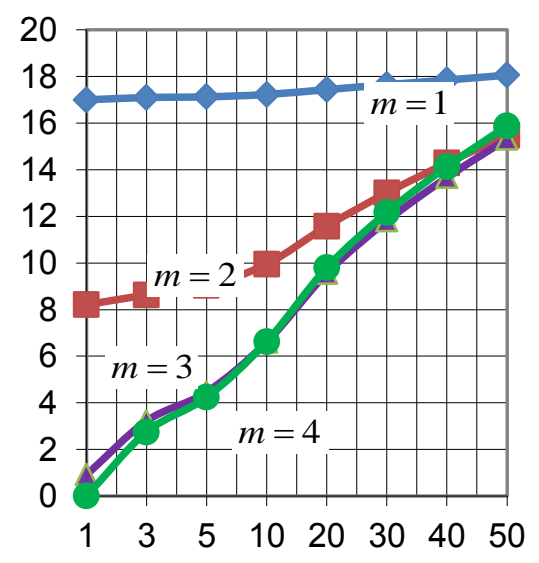

Fig. 4 - Dependence of frequencies of free oscillations of a semi-underground PE 100 gas pipeline on the value of the coefficient of the elastic medium $k$, with $n=\mathbf{4}$

As a result of oscillations, the cross section of the pipeline is deformed, but it strongly depends on the geometrical and mechanical parameters set, for example, on the stiffness coefficient of the elastic medium $\mathrm{k}$.

The smallest frequencies of free oscillations of a polyethylene pipeline are of practical interest in the work, since it is for the three lowest frequencies that the entire system is detuned from resonance. In this regard, we will continue to consider only the lowest frequencies.

The analysis of the obtained frequency values showed that the minimum frequencies $\omega_{m n}$ will significantly depend on the value of the stiffness coefficient of the elastic medium. With an increase in the values of this coefficient, and depending on the form of the oscillations, the frequencies can increase by, about $80 \%$. This is due to an increase in the stiffness of the soil around the pipeline, and as a result of an increase in the rigidity of the pipeline itself. It should be noted that due to the increase in the stiffness coefficient of the elastic medium, the minimum frequencies will depend on the mode of oscillation, for example, at $\mathrm{k}=3 \mathrm{MN} / \mathrm{m}^{3}$ (fresh sand) minimum frequencies are realized with $\omega_{44}=$ $2,75 \mathrm{~Hz}$, which is accompanied by the deformation of the cross section of the pipeline, and the formation of four half-waves in the circumferential and four in the longitudinal direction, and at $\mathrm{k}=50 \mathrm{MN} / \mathrm{m}^{3}$ (soils are dense) $\omega_{21}=10,9 \Gamma u$ (one half-wave in the circumferential and in the longitudinal direction). In the latter case, the work of the pipeline resembles the work of a thin-walled rod, without taking into account the deformation of the cross section.

\section{References}

1 F. Li, B. Wei, X. Zhao, X. Shao, R. Cai, Trenchless Technology - The Best Choice for Underground Pipeline Construction and Renewal, Proceedings of the International Conference on Pipelines and Trenchless Technology (2013) 
2 E.L. Aksel'rad, V.P. Il'in, Raschet truboprovodov (Mashinostroenie, 1972)

3 V.G. Sokolov, I.O. Razov, Vest. grazhd. Inzh. 6 (2016)

4 V.G. Sokolov, Kolebaniya, staticheskaya i dinamicheskaya ustoychivost' truboprovodov bol'shogo diametra: dis. doktor. tekhn. Nauk (Spb, 2011)

5 V.G. Sokolov, Prom.grajd.stroit 8 (2010) 